\title{
A Simple Ultrasensitive Displacement Sensor Based on a High Bend Loss Single-Mode Fibre and a Ratiometric Measurement System
}

\author{
Pengfei Wang \\ Technological University Dublin, pengfei.wang@tudublin.ie \\ Gilberto Brambilla \\ University of Southampton \\ Yuliya Semenova \\ Technological University Dublin, yuliya.semenova@tudublin.ie
}

See next page for additional authors

Follow this and additional works at: https://arrow.tudublin.ie/engscheceart

Part of the Electromagnetics and Photonics Commons

\section{Recommended Citation}

Wang, P., Brambilla, G., Semenova, Y., Wu, Q., Farrell, G.: A Simple Ultrasensitive Displacement Sensor Based on a High Bend Loss Single-Mode Fibre and a Ratiometric Measurement System. Journal of Optics, Vol.13, 7, 2011, article no. 075402. doi:10.1088/2040-8978/13/7/075402

This Article is brought to you for free and open access by the School of Electrical and Electronic Engineering at ARROW@TU Dublin. It has been accepted for inclusion in Articles by an authorized administrator of ARROW@TU Dublin. For more information, please contact arrow.admin@tudublin.ie, aisling.coyne@tudublin.ie, gerard.connolly@tudublin.ie.

Funder: IRCSET, Marie Curie Actions under FP7, Royal Society London, SFI

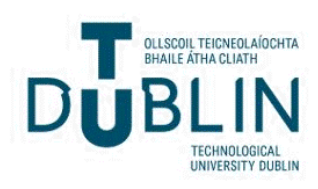




\section{Authors}

Pengfei Wang, Gilberto Brambilla, Yuliya Semenova, Qiang Wu, and Gerald Farrell 


\section{A simple ultrasensitive displacement sensor based on a high bend loss single-mode fibre and a ratiometric measurement system}

Q.1 Pengfei Wang ${ }^{1,2,3}$, Gilberto Brambilla ${ }^{1}$, Yuliya Semenova ${ }^{2}$, Qiang $\mathrm{Wu}^{2}$ and Gerald Farrell ${ }^{2}$

\footnotetext{
${ }^{1}$ Optoelectronics Research Centre, University of Southampton, Southampton SO17 1BJ, UK

${ }^{2}$ Photonics Research Centre, School of Electronic and Communications Engineering, Dublin Institute of Technology, Kevin Street, Dublin 8, Ireland

E-mail: pw3y09@orc.soton.ac.uk
}

Received 4 January 2011, accepted for publication 29 March 2011

Published

Online at stacks.iop.org/JOpt/13/000000

\begin{abstract}
An all-fibre displacement sensor with a simple configuration and capable of monitoring sub-50 nm displacements is proposed and investigated experimentally. The proposed fibre displacement sensor consists of a half-loop structure of bare high bend loss single-mode fibre 1060XP and a ratiometric power interrogation system. By measuring the change in transmission ratio in the ratiometric system, a change in displacement can be measured. The displacement sensor is sensitive to temperature and an experimental investigation of this sensitivity is presented. It is found that the peak shift response has a linear variation with temperature; therefore, temperature dependence can be mitigated by a suitable displacement correction process. The proposed macrobending fibre based displacement sensor benefits from simplicity and low cost and achieves a comparable resolution as compared with other conventional fibre optic sensors.
\end{abstract}

Keywords: fibre optic sensor, displacement sensor, bend loss, ratiometric

(Some figures in this article are in colour only in the electronic version)

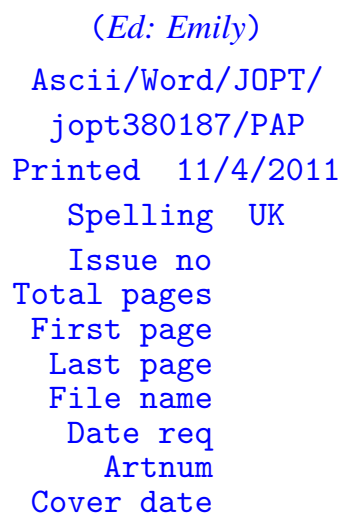

(Ed: Emily)

scii/Word/JOPT/

Spelling UK

Last page

ile name

Cover date

\section{Introduction}

Q.3 Optical fibre can be used in a number of ways as a sensing element. Fibre sensors offer advantages such as immunity to electromagnetic interference and ease of fabrication. Recently, several kinds of optical fibre sensors have been proposed for applications in growth areas for sensing [1-4], such as building structural monitoring, bearing analysis, fatigue testing, variation and microposition sensing.

Existing fibre based displacement sensors have many advantages, but such advantages are often offset by the complexity of the interrogation system. For example fibre Bragg grating (FBG) sensors [5-8] are used frequently for

\footnotetext{
3 Author to whom any correspondence should be addressed.
}

displacement sensing but have the disadvantage that the interrogation system for extracting the displacement value is complex, as the displacement is measured by estimating a small wavelength shift.

Previous work undertaken by us has shown that macrobending loss can be utilized in edge filter, temperature and refractive index sensing applications [9-11]. In particular, in the published work $[9,12]$, both theoretical and experiment results have shown that the fibre bend diameter alters the bend loss significantly. It is this characteristic that offers the possibility to develop a novel displacement sensor based on a macrobending structure. Therefore, in this paper, the approach proposed will utilize the macrobending loss in a single-mode optical fibre loop to sense displacement. Since optical fibre bend loss is used to determine displacement it is possible to use 
an interrogation system based on a simple ratiometric optical power measurement technique and a fixed single-wavelength source.

The investigation of the macrobending fibre based displacement sensor includes: (1) the displacement sensing principle; (2) the structure of the proposed sensor device; (3) the fabrication and performance of the proposed displacement sensor. A ratiometric interrogation system for the sensor is also investigated. The proposed macrobending fibre based displacement sensor provides a high resolution (circa $40 \mathrm{~nm}$ ) and, in contrast with existing FBG based sensors, also offers the advantages of a much simpler configuration, ease of fabrication and low cost.

\section{Design of the fibre displacement sensor}

In our previous work [9], a bare 1060XP fibre (Nufern) coated with an absorbing layer has been developed as an edge filter for wavelength measurement applications. Both theoretical and experimental results have shown that the bend loss monotonically decreases as the bend diameter increases at a fixed wavelength. Such a characteristic offers a possibility to develop a macrobending based displacement sensor. The operating principle of such a sensor is that as the bend diameter of the fibre section is changed, the bending loss changes. By measuring the changes in macrobending loss, the variation of bend diameter can be measured and with a suitable calibration the displacement can be determined.

However, using the bare 1060XP fibre coated with an absorbing layer in [9] as the basis of a displacement sensor would result in low resolution for the proposed sensor. From [12] which also utilizes a 1060XP fibre, one can see that the measured discrimination range (bend loss difference between 8.5 and $14 \mathrm{~mm}$ of bend radius) is $15.5 \mathrm{~dB}$, at an operating wavelength of $1500 \mathrm{~nm}$, which yields an estimated

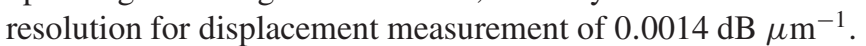
Given that low cost optical power measurement normally cannot achieve a resolution better than $0.01 \mathrm{~dB}$, micron level displacement sensing cannot be achieved using this fibre configuration. Furthermore the limitations of the absorbing layer applied on the fibre surface, evident from the quasiperiodic behaviour of the measured results, can further affect the precision of the displacement measurement. For example, the discrepancy between the measured results and theoretical model has been presented as circa $1.6 \mathrm{~dB}$ in [12], which would mean an unacceptable variation of $1142.86 \mu \mathrm{m}$ in terms of displacement measurement.

One possible solution for increasing the sensitivity of the proposed macrobending fibre sensor is to utilize the inherent interference effects in the cladding. References [13, 14] have investigated a bare bending SMF28 fibre, where strong interference significantly affects the light propagation performance. The measured bend loss results show a large discrimination range $(\sim 7.3 \mathrm{~dB})$ between a bend radius of $6 \mathrm{~mm}$ and one of $5.5 \mathrm{~mm}$, at a wavelength of $1600 \mathrm{~nm} \mathrm{[13].}$ This performance indicates that such interference could be potentially useful for enhancing the sensitivity of displacement sensing using a macrobending loop. However because of the

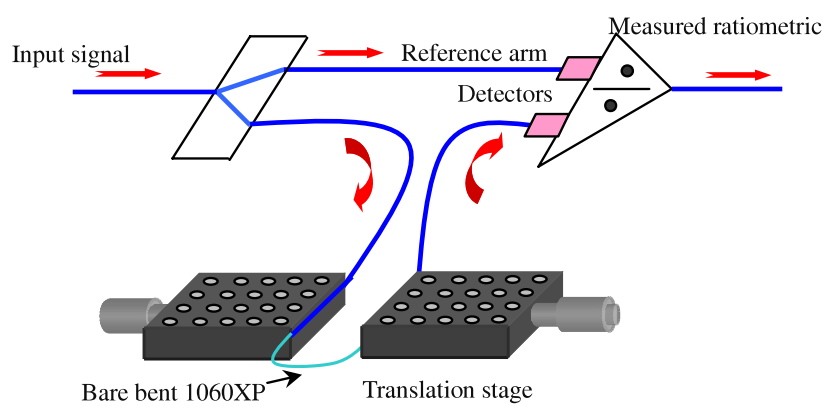

Figure 1. Experimental setup of the ratiometric measurement system which involves the proposed displacement sensor.

low bend loss at 1500-1600 nm of the SMF28, a displacement sensor using SMF28 would require many fibre turns to achieve a reasonable resolution, which in turn would create mechanical difficulties for the sensor in practice.

The alternative is to use a high bend loss fibre, allowing a sensor to be fabricated with only a half-loop of fibre. In this paper, the proposed fibre displacement sensor consists of a half-loop structure of bare high bend loss fibre (the coating has been stripped). The fibre employed in the experiment was a 1060XP single-mode fibre which has a diameter of $125 \mu \mathrm{m}$ and a high bend loss over the wavelength range of 1500-1600 nm. Experimentally, to fix the diameter of the fibre half-loop, each end of the half-loop is glued to a $20 \mathrm{~mm}$ travel single-axis translation stage, which can achieve a $10 \mu \mathrm{m}$ step resolution. In this way the diameter of the bend fibre can be controlled using the translation stages.

The displacement variation can be extracted from a measurement of the bend loss, which in turn can be found using a simple ratiometric power measurement system, as is shown in figure 1. A ratiometric system is made in the lab and used, as it provides independence from source power changes resulting in a more stable and accurate system. As shown in figure 1, the schematic structure of a ratiometric measurement system includes a splitter, a fibre sensor, a reference arm and two photodetectors. The fibre sensor discriminates between the power oscillations of the input signal affected by the surrounding measurands with the transmission measured by the upper photodetector. The reference arm is based on the ratio between the measured powers from two arms, it can discriminate between the oscillations of the input signal regardless of its power level for an ideal input light (monochromatic) and photodetectors (no noise). The input signal from the source is split into two equal signals; one passes through the fibre sensor and the other goes to the reference arm. Two photodiodes are placed at the ends of both arms to measure the output power. By measuring the ratio of the two output signals, which is a function of the fibre bending diameter, the displacement can be measured, assuming that a suitable calibration has taken place.

It is well known that for the bare bending fibre, the effect of the reflection of the radiated field at the interface between the cladding layer and air is critical and the reflection depends 


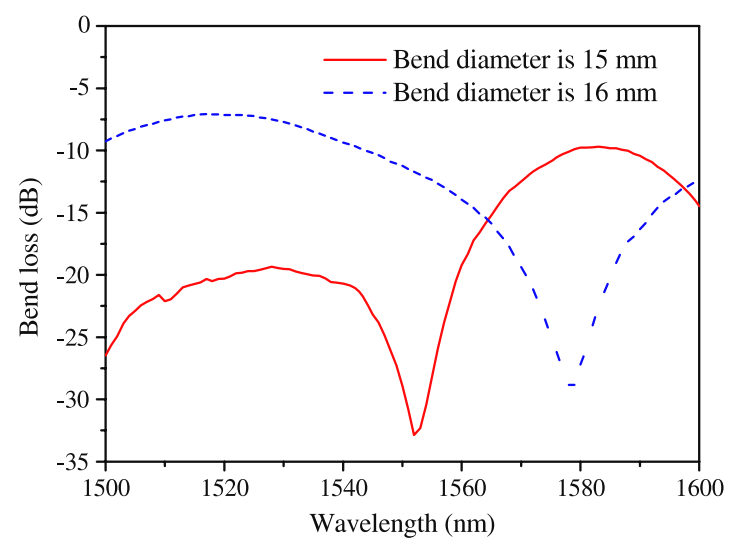

Figure 2. Measured bend loss of bare 1060XP fibre at the bend diameter of 15 and $16 \mathrm{~mm}$, respectively.

on the outside conditions and thus has a significant influence on the intensity of interferences in the region of fibre cladding. In order to refine the roughness of the surface of fibre cladding, the polymer coating layers of 1060XP fibre were stripped using hot concentrated sulfuric acid $\left(\mathrm{H}_{2} \mathrm{SO}_{4},>95 \mathrm{wt} \%\right.$, at a temperature of about $200^{\circ} \mathrm{C}$ ). After the chemical stripping process, we measured the bend loss of the bare 1060XP fibre in the wavelength range from 1500 to $1600 \mathrm{~nm}$ using a broadband optical source and an optical spectrum analyser, for bend diameters of $15-16 \mathrm{~mm}$ (the bend length is a halfturn). The relationship between bending loss and radius over a wavelength range of $1500-1600 \mathrm{~nm}$ is presented in figure 2 for bend diameters of 15 and $16 \mathrm{~mm}$. It is evident from figure 2 that for a given bend diameter there is a complex relationship between wavelength and bend loss which arises because of reflections of the radiated field at the interface between the cladding layer and air. The reflected light interferes with the propagating fundamental mode within the core. At a single wavelength the resulting interference means that the bending loss is a strong function of the bend diameter. For example for a wavelength of $1550 \mathrm{~nm}$, one can see that the difference in bend loss between 15 and $16 \mathrm{~mm}$ is over $17.7 \mathrm{~dB}$, which will provide a useful displacement resolution. Note that it is found that beyond $18 \mathrm{~mm}$ the effect of interference is too low to provide a useful sensitivity enhancement, while below $15 \mathrm{~mm}$, the risk of fibre breakage by excessive stress is considerable.

In the experiments with the ratiometric measurement system, the ratio response was measured at $10 \mu \mathrm{m}$ intervals for a bend diameter range of 15000-15260 $\mu \mathrm{m}$. A tunable laser EXFO FLS-2600B with an output light at a wavelength of $1550 \mathrm{~nm}$ and a wavelength stability of $< \pm 6 \mathrm{pm}$ (after $1 \mathrm{~h}$ warming up) was used as the input signal source. The measured ratio responses are presented in figure 3(a), and a polynomial fit is also given in the figure. From the figure, it is clear that the measured ratio response decreases monotonically as the bend diameter increases, and one can also see that the maximum changes in the ratio response occur at small radii/displacements.

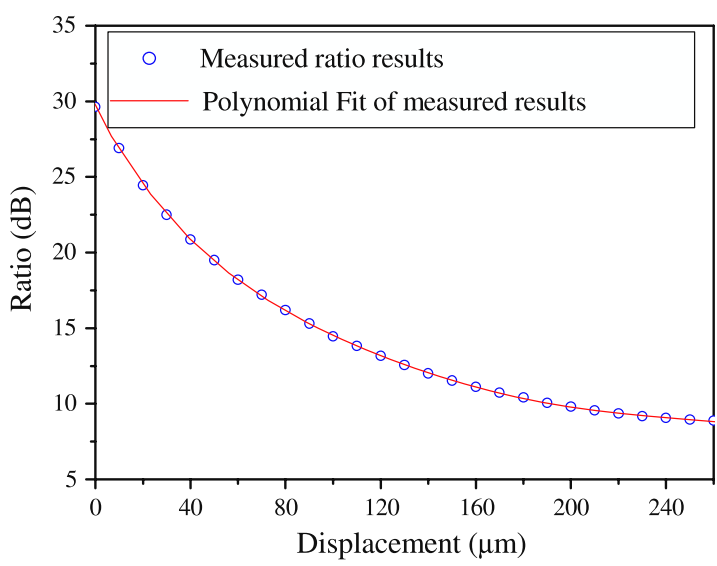

(a)

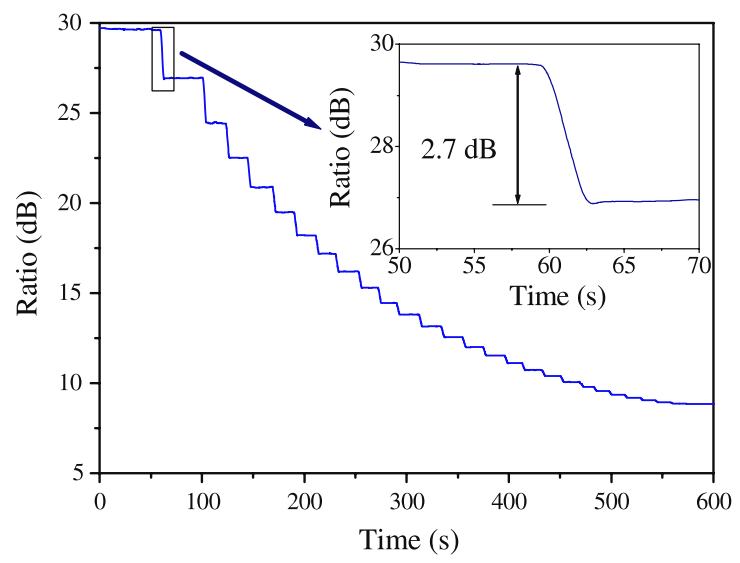

(b)

Figure 3. (a) Bend loss ratio response as a function of displacement; (b) variation in ratio for a displacement interval of $10 \mu \mathrm{m}$ for the bend radius of $15-15.26 \mathrm{~mm}$.

\section{Measurement of the resolution of the proposed fibre displacement sensor}

To measure the displacement resolution of the ratiometric measurement system, the bending radius is changed from 15000 to $15260 \mu \mathrm{m}$ with an incremental step change of $10 \mu \mathrm{m}$ over a time period of $600 \mathrm{~s}$. The corresponding measured ratio variation is shown in figure 3(b), which proves that the system is very much capable of resolving micron level displacement changes. From the figure, one can see that the resolution at the position of $15000-15010 \mu \mathrm{m}$ is circa $0.27 \mathrm{~dB} \mu \mathrm{m}^{-1}$. Since the minimal value of ratio variation detectable is $\sim 0.01 \mathrm{~dB}$, a displacement smaller than $50 \mathrm{~nm}$ can be measured over this displacement range using the ratiometric system.

To verify the resolution assumed above, a commercial PZT stack AE0505D18 (manufactured by Tokin Corporation, Japan) was used as the transducer. It has a length of $20 \mathrm{~mm}$ and cross section dimensions of $5 \mathrm{~mm} \times 5 \mathrm{~mm}$, and can produce the maximum elongation of $15 \mu \mathrm{m}$ at a DC voltage of $100 \mathrm{~V}$. The bare high bend loss fibre 1060XP was fixed as the displacement sensor head. As shown in figure 4 the bare bend fibre is glued to the platform and the base, with a total length in between the fixed points of about $15 \mathrm{~mm}$. 


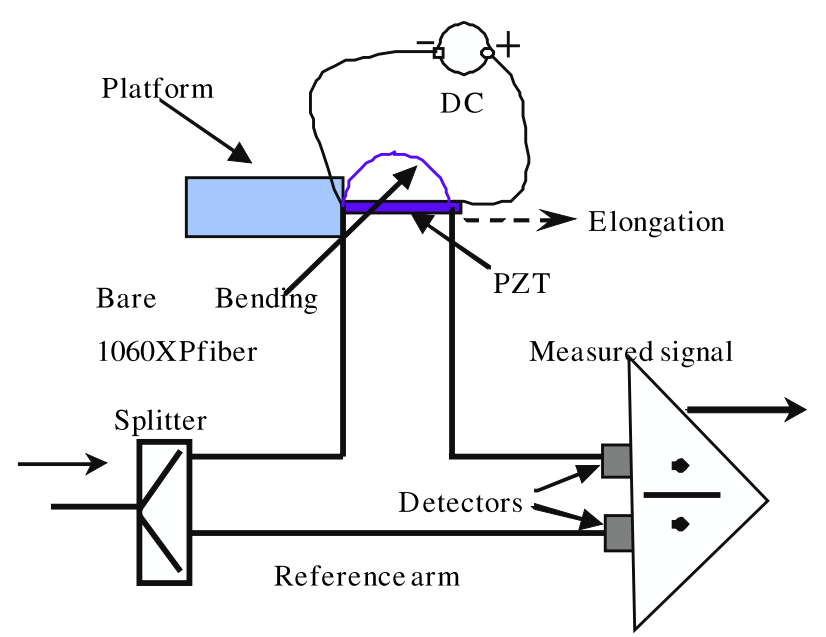

Figure 4. Schematic configuration of the proposed bent fibre voltage sensor system.

A tunable laser EXFO FLS-2600B was used as the input signal source. A single-channel piezocontroller MDT694A (Thorlabs) was used to supply the DC voltage from 0 to $100 \mathrm{~V}$ to the PZT stack. Figure 5(a) shows the measured ratio response at the wavelength of $1550 \mathrm{~nm}$ versus submicron displacement for increments of $0.75 \mu \mathrm{m}$. The ratio response exhibits a linear dependence on the displacement with a slope of $0.268 \mathrm{~dB} \mu \mathrm{m}^{-1}$. To estimate the displacement measurement resolution of the system, step changes of $75 \mathrm{~nm}$ starting from the bend diameter of $15006 \mu \mathrm{m}$ are applied to the fibre displacement sensor with time intervals of $5 \mathrm{~s}$. The measured ratio variations versus time are shown in figure $5(\mathrm{~b})$. It is clear that the resolution of the displacement sensing system is better than $50 \mathrm{~nm}$. Thus it is demonstrated that the proposed fibre displacement sensor with a macrobending fibre structure is suitable for displacement measurements with a reasonably high resolution of $\sim 40 \mathrm{~nm}$.

\section{Temperature-induced variations in the displacement sensing system}

Actually in real-world fibre optic sensing applications, temperature effects are well known to have a significant influence on the properties of a fibre optic sensor, given both thermo-optic and thermal expansion effects of fibre materials. Therefore, it is worthwhile and necessary to investigate the temperature-dependent behaviour of the proposed fibre displacement sensor. Experiments were carried out for the $15 \mathrm{~mm}$ bend diameters to investigate the temperature dependence of the displacement sensor. To avoid the experimental variations induced by thermal expansion of the metallic aluminium translation stages, the macrobending fibre sensor head with a half-loop structure was fixed on a hollow plastic polyvinyl chloride tube with a precise diameter of $15 \mathrm{~mm}$ and placed into a temperature-controlled heating oven. A reference ratio response of the system was obtained at room temperature $\left(20^{\circ} \mathrm{C}\right)$. The thermal expansion coefficient of polyvinyl chloride plastic material is $\sim 7 \times 10^{-5}{ }^{\circ} \mathrm{C}^{-1}$ and the plastic tubes are $1 \mathrm{~mm}$ in thickness; therefore, the

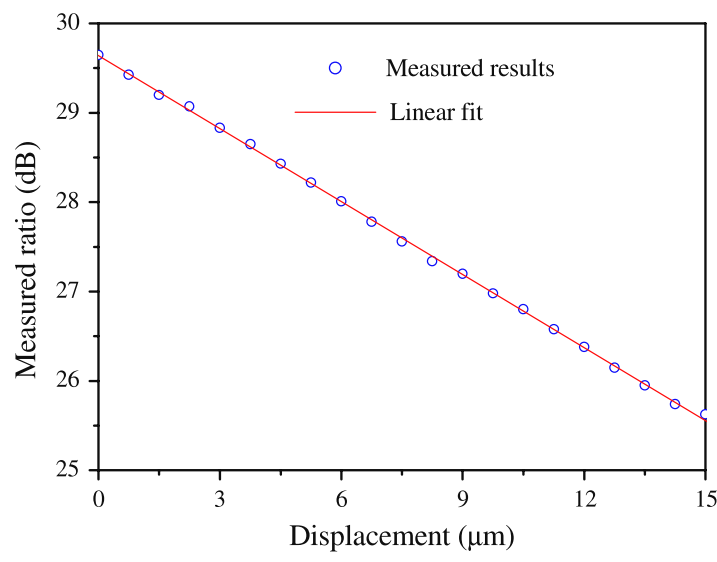

(a)

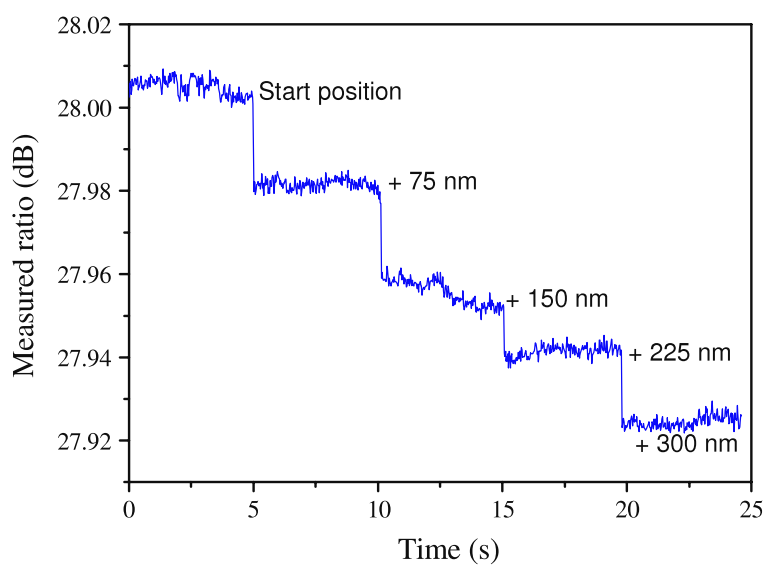

(b)

Figure 5. (a) Measured ratio response of the sensor against displacement $(0-15 \mu \mathrm{m})$ at the operating wavelength of $1550 \mathrm{~nm}$ (variation in ratio for a step change of $0.75 \mu \mathrm{m}$, which corresponds to a $5 \mathrm{~V}$ voltage step applied to the PZT); (b) measured output ratio response of the sensor versus time (0-25 s) at the operating wavelength of $1550 \mathrm{~nm}$ (variation in ratio for a step displacement change of $75 \mathrm{~nm}$ corresponding to a step voltage change of $0.5 \mathrm{~V}$ ).

thermal expansion rate of the rods is $\sim 70 \mathrm{~nm}{ }^{\circ} \mathrm{C}^{-1}$, and the influence of the thermal expansion of the plastic rods can be neglected in this experiment. The variation in the spectral responses from the reference response at $20^{\circ} \mathrm{C}$ was measured for a temperature range of $20-30^{\circ} \mathrm{C}$ with an interval of $0.5^{\circ} \mathrm{C}$. The dependence of the ratiometric response on temperature for the bend diameter of $15 \mathrm{~mm}$ is shown in figure 6. The measured average slope of the resonance peak shift is $0.096 \mathrm{~nm}{ }^{\circ} \mathrm{C}^{-1}$. This result shows that the sensor has a rather strong temperature dependence. This temperature-dependent resonance peak shift is expected and it has been discussed in previous published work [10, 11]. However, since the peak shift monotonically decreases with the temperature, it is possible to apply a correction factor to mitigate the temperature-induced errors. To verify this, the required temperature correction for the ratio response, which is effectively a correction factor for displacement, is calculated using the polynomial fit presented in figure 3(a) and shown in figure 6 for different temperatures with an interval of $0.5^{\circ} \mathrm{C}$ in the range from 20 to $30^{\circ} \mathrm{C}$. 


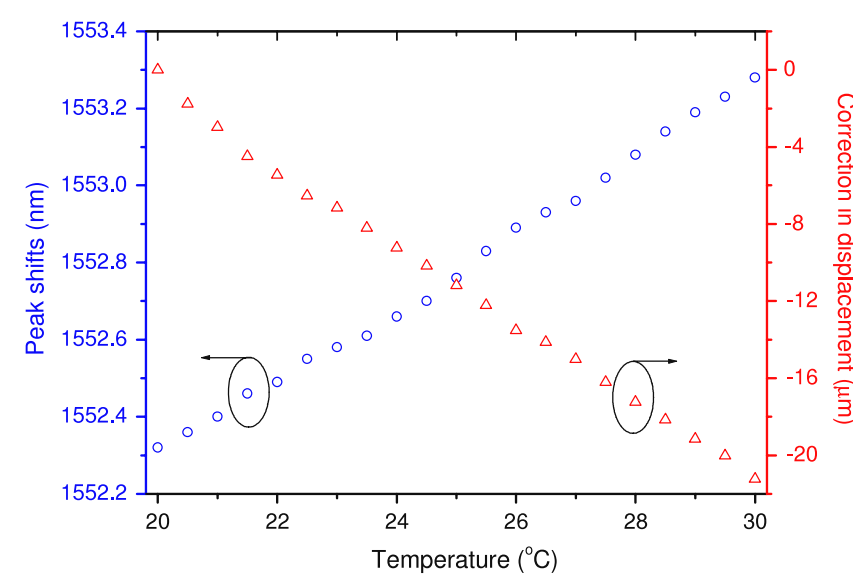

Figure 6. Resonance peak shifts and corresponding correction in displacement as a function of temperature variation when the bent diameter is $15 \mathrm{~mm}$.

\section{Conclusion}

To conclude, an all-fibre submicron displacement sensor with a simple optical configuration has been developed. The fibre displacement sensor presented consists of a half-loop structure of coating stripped bare 1060XP single-mode fibre, employing the interference effect in the fibre cladding region to improve sensitivity. A ratiometric system involving the sensor has been developed, and corresponding results have been presented, which have shown a better than $50 \mathrm{~nm}$ resolution on displacement, verified by experimental results. The temperature-induced variations in resonance peak shifts and displacement have been investigated and presented in this article. Compared with a conventional FBG displacement sensor, the macrobending bare fibre based sensor developed has shown a comparable resolution performance and does not require a complex fabrication. The displacement sensor presented in the paper can be used to measure a range of measurands such as displacement, vibration and strain for indoor applications, for example in optical microphones, indoor mobile robot odometry measurements and telerobotic strain/force sensing for microsurgery application etc.

\section{Acknowledgments}

P Wang is funded by the Irish Research Council for Science, Engineering and Technology, co-funded by the Marie Curie Actions under FP7. G Brambilla gratefully acknowledges the
Royal Society (London, UK) for his University Research Fellowship. Q Wu gratefully acknowledges the support of Science Foundation Ireland under grant no. 07/SK/I1200.

\section{References}

[1] Puangmali P, Althoefer K and Seneviratne L D 2010 Mathematical modeling of intensity-modulated bent-tip optical fiber displacement sensor IEEE Trans. Instrum. Meas. $59283-91$

[2] Allen G, Sun K-X and Byer R 2010 Fiber-coupled, Littrow-grating cavity displacement sensor 35 1260-2

[3] Yang H Z, Lim K S, Harun S W, Dimyati K and Ahmad H 2010 Enhanced bundle fiber displacement sensor based on concave mirror Sensors Actuators A 162 8-12

[4] Wu Q, Semenova Y, Wang P, Hatta A and Farrell G 2011 Experimental demonstration of a simple displacement sensor based on a bent single-mode-multimode-single-mode fiber structure Meas. Sci. Technol. 22025203

[5] Hsu C C, Wu C C, Lee J Y, Chen H Y and Weng H F 2008 Reflection type heterodyne grating interferometry for in-plane displacement measurement Opt. Commun. 2812582

[6] Ma C C and Chuang K C 2008 Investigation of the transient behavior of a cantilever using a point-wise fiber Bragg grating displacement sensor system Smart Mater. Struct. 17065010

[7] Chuang K-C and Ma C-C 2010 Multidimensional dynamic displacement and strain measurement using an intensity demodulation-based fiber Bragg grating sensing system J. Lightwave Technol. 28 1897-905

[8] Kim H, Yoon J-S, Kim H-B and Han J-H 2010 Measurement of the thermal expansion of space structures using fiber Bragg grating sensors and displacement measuring interferometers Meas. Sci. Technol. 21085704

[9] Wang P, Farrell G, Wang Q and Rajan G 2007 An optimized macrobending-fiber-based edge filter IEEE Photon. Technol. Lett. 19 1136-8

[10] Wang P, Rajan G, Farrell G and Semenova Y 2008 Temperature dependence of a macrobending edge filter based on a high-bend loss fiber Opt. Lett. 33 2470-2

[11] Wang P, Semenova Y, Wu Q, Farrell G, Ti Y and Zheng J 2009 Macrobending single-mode fiber-based refractometer Appl. Opt. 48 6044-9

[12] Wang P, Farrell G, Semenova Y and Rajan G 2008 Influence of fiber manufacturing tolerances on the spectral response of a bend loss based all-fiber edge filter Appl. Opt. 47 2921-5

[13] Wang P, Wang Q, Farrell G, Rajan G, Freir T and Cassidy J 2007 Investigation of macrobending losses of standard single mode fiber with small bend radii Microw. Opt. Technol. Lett. $492133-8$

[14] Nam S H and Yin S 2005 High-temperature sensing using whispering gallery mode resonance in bent optical fibers IEEE Photon. Technol. Lett. 17 2391-3 


\section{Queries for IOP paper 380187}

\section{Journal: JOpt \\ Author: $\quad$ P Wang et al}

Short title: A simple ultrasensitive displacement sensor based on a high bend loss single-mode fibre and a ratiometric measurement system

Page 1

Query 1:

Author: Please check the author names and affiliations carefully.

Query 2:

Author: Please be aware that the colour figures in this proof will normally only appear in colour in the online Web version. If you require colour in the printed journal and have not previously arranged it, please contact the Publishing Administrator now.

Query 3:-

Author: In several places I have standardized the English somewhat. Please check that I have not inadvertently altered the meaning.

\section{Page 5}

Query 4:-

Author: Please check the details for any journal references that do not have a blue link as they may contain some incorrect information. Pale purple links are used for references to arXiv e-prints.

Query 5:-

Author: [2]: Please provide journal title. 